\title{
Attenuated acute salivary $\alpha$-amylase responses to gustatory stimulation with citric acid in thin children
}

\author{
Long Hui Chen ${ }^{1,2}$, Ze Min Yang ${ }^{2 *}$, Wei Wen Chen ${ }^{1 *}$, Jing Lin $^{2}$, Min Zhang ${ }^{3}$, Xiao Rong Yang ${ }^{4}$ and \\ Ling Bo Zhao 5 \\ ${ }^{1} \mathrm{Pi}$-Wei Institute, Guangzhou University of Chinese Medicine, Guangzhou, People's Republic of China \\ ${ }^{2}$ School of Basic Courses, Guangdong Pharmaceutical University, Guangzhou, People's Republic of China \\ ${ }^{3}$ Haizhu Maternal and Child Health Hospital, Guangzhou, People's Republic of China \\ ${ }^{4}$ The First Affiliated Hospital/School of Clinical Medicine of Guangdong Pharmaceutical University, Guangzhou, \\ People's Republic of China \\ ${ }^{5}$ Clinical Medical College of Acupuncture, Moxibustion and Rehabilitation, Guangzhou University of Chinese Medicine, \\ Guangzhou, People's Republic of China
}

(Submitted 1 May 2014 - Final revision received 25 December 2014 - Accepted 21 January 2015 - First published online 18 March 2015)

\begin{abstract}
Salivary $\alpha$-amylase (SAA) is responsible for the 'pre-digestion' of starch in the oral cavity and accounts for up to $50 \%$ of salivary protein in human saliva. An accumulating body of literature suggests that sAA is of nutritional importance; however, it is still not clear how sAA is related to individual's nutritional status. Although copy number variations (CNV) of the salivary amylase gene (AMY1) are associated with variation in sAA levels, a significant amount of sAA variation is not explained by $A M Y 1 \mathrm{CNV}$. To measure sAA responses to gustatory stimulation with citric acid, we used sAA ratio (the ratio of stimulated sAA levels to those of resting sAA) and investigated acute sAA responses to citric acid in children with normal (Normal-BMI, $n$ 22) and low (Low-BMI, $n$ 21) BMI. The AMY1 gene copy number was determined by quantitative PCR. We, for the first time, demonstrated attenuated acute sAA responses (decreased sAA ratio) to gustatory stimulation in Low-BMI (thinness grade 3) children compared with the Normal-BMI children, which suggest that sAA responses to gustatory stimulation may be of nutritional importance. However, child's nutritional status was not directly related to their resting or stimulated sAA levels, and it was not associated with $A M Y 1$ gene copy number. Finally, $A M Y 1 \mathrm{CNV}$ might influence, but did not eventually determine, sAA levels in children.
\end{abstract}

Key words: Salivary $\alpha$-amylase: Acute responses: Children: BMI: Salivary amylase gene: Nutritional status

Salivary $\alpha$-amylase (sAA), which is routinely categorised as resting (unstimulated) or stimulated, is the most abundant protein in human saliva ${ }^{(1)}$, accounting for up to $50 \%$ of sali${\text { vary } \text { protein }^{(2)} \text {, and is responsible for starch hydrolysis }}^{(3)}$. Genetically, sAA production is influenced by individual copy number variations (CNV) of the salivary amylase gene ( $A M Y 1$ ), which codes for sAA ${ }^{(4)}$. sAA concentrations positively correlate with the copy number of the $A M Y 1$ gene in adults $^{(5,6)}$. However, a significant amount of variation in SAA levels is not explained by $A M Y 1 \mathrm{CNV}^{(5,6)}$. The $A M Y 1$ gene shows extensive variation in copy number ${ }^{(7,8)}$, with a range of anywhere from two to fifteen diploid copies. sAA levels considerably differ between populations with high-starch diets and those with low-starch diets ${ }^{(5)}$. The patterns of between-population differences have been linked to the CNV of $A M Y 1$ gene and seen as an adaptive response to the intake of dietary starch on an evolutionary time scale ${ }^{(5)}$. In addition to gene-dependent variation, differences in sAA levels have been detected in response to various physiological and psychosocial stress conditions ${ }^{(9-11)}$. For example, food intake (mastication) can induce dramatic increase in sAA levels ${ }^{(12,13)}$. In addition, gustatory stimulation (by application of citric acid) also stimulates pronounced increase in sAA levels $^{(14)}$.

sAA is known to be mainly involved in the initiation of the digestion of starch in the oral cavity. However, the nutritional advantage provided by the amylolytic 'pre-digestion' of starch in the oral cavity has rarely been established, since the

Abbreviations: $A M Y 1$, salivary amylase gene; CNV, copy number variations; CPIR, cephalic-phase insulin release; qPCR, quantitative PCR; sAA, salivary $\alpha$-amylase. 
majority of ingested starch is digested in the small intestine by pancreatic amylase. Several studies, although, have linked sAA levels with the oral perception of textural attributes of starchy foods ${ }^{(6,15,16)}$, which can explain how sAA benefits nutrition. In a previous study, custards with added $\alpha$-amylase resulted in increased melting and decreased thickness sensations in the mouth of human subjects, while the opposite effects were observed when custards were added with acarbose (an amylase inhibitor). Then, the authors argued that the effects of amylase on perceived melting and thickness were caused by amylase-induced breakdown of starch ${ }^{(15)}$. Another study has found that subjects with high sAA activity have lower perceived thickness for the starch-based custard and experience decreased creamy after feel in both custard and mayonnaise ${ }^{(16)}$. More recently, Mandel et al. ${ }^{(6)}$ successfully linked sAA level with the oral perception of starch by introducing time-intensity ratings to track the digestion of starch during oral manipulation. In their study, individuals with high sAA levels reported faster and more significant decreases in perceived starch viscosity than did individuals with low sAA levels ${ }^{(6)}$. Oral changes in viscosity, or thickness, play an important role in determining individual's liking and preerence for food ${ }^{(17)}$. Collectively, these data suggest that the amylolytic 'pre-digestion' of starch-based foods by sAA may be nutritionally important, by influencing the oral perception of textural attributes of starchy foods and thus determining an individual's liking and preference for a starchy food at the preprandial stage, especially for those who traditionally feed on starch-rich foods. Interestingly, Mandel \& Breslin ${ }^{(18)}$ raised another possibility that this 'pre-digestion' may also benefit nutrition by influencing the plasma insulin concentrations and blood glucose levels at the postprandial stage.

To date, however, few data are available on the direct relationships between sAA levels and individual's nutritional status. It is difficult, although, to link sAA levels with individual's nutritional status directly by the facts that individuals are accustomed to their own idiosyncratic salivary flow rates and sAA levels, and that sAA levels show high variability among and within individuals. More parameters need to be introduced to explain how sAA relates to individual's nutritional status. SAA ratio is a ratio of the stimulated sAA levels to that of resting SAA (stimulated/resting), which reflects the extent to which sAA responds to stimulation. We hypothesised that sAA ratio may be related to individual's nutritional status.

Childhood is a sensitive period of growth and development, which makes children more susceptible to environmental changes than adults. For instance, changes in oral perception, which are associated with sAA levels ${ }^{(6,15,16)}$, may significantly influence child's liking and preference for foods, and thus play a crucial role in determining their nutritional status, while in adults, it is already shaped by combined actions of innate and environmental factors. We expected that it would be difficult to find direct connections between sAA levels and their nutritional status. Children are expected to have more similar but less psychosocial stress conditions than adults, which would serve to introduce less variations in sAA levels since these stress factors significantly influence individual sAA levels, and adults have unmanageable and varying psychosocial stress conditions. In addition, basal sAA activity in children has reached adult levels ${ }^{(19)}$, and acute sAA responses have also been found in children ${ }^{(20)}$. Based on these considerations, in the present study, we investigated whether nutritional status in children is related to (1) sAA levels and (2) $A M Y 1$ gene copy number. The present study did not include overweight or obese children, since they were shown to have sAA levels similar to those of normal-weight children ${ }^{(21)}$.

\section{Materials and methods}

\section{Ethics statement}

The present study was conducted according to the guidelines laid down in the declaration of Helsinki, and all procedures involving human subjects/patients were approved by the Academic Ethics Committee of Guangzhou University of Chinese Medicine. Written informed consent was obtained from all subjects/patients. All participants (children) were accompanied by at least one of their parents, who also signed the informed consent and stayed throughout the study.

\section{Participants}

Children aged 5-12 years were recruited in Haizhu Maternal and Child Health Hospital. We included Low-BMI children (thinness grade 3) based on BMI cut-offs, which were defined by international survey of six large nationally representative cross-sectional studies ${ }^{(22)}$, and Normal-BMI children. Overweight or obese children were excluded based on the criteria established by the Working Group on Obesity in China ${ }^{(23)}$. Individuals using asthma medications, psychotropic substances or painkillers were excluded. Participants were free of psychiatric, sever somatic and oral diseases, as evaluated from an interview by one of the authors (M. Z.). Use of vitamins and natural therapeutics was allowed, and participants were instructed to take their prescribed drugs at least $5 \mathrm{~h}$ before intervention. Individuals who reported acute caffeine consumption were excluded, because caffeine ingestion leads to significant increases of sAA activity and output ${ }^{(24)}$. Participants were not allowed to eat or drink (anything but water) or to take strenuous exercise at least $1 \mathrm{~h}$ before intervention, because these factors significantly modify sAA activity $^{(19)}$. Forty-three children (twenty-three boys and twenty girls) met the inclusion criteria. Of them, twenty-two children (thirteen boys and nine girls) were assigned to the normal-BMI group (healthy) and the remaining twenty-one children (ten boys and eleven girls) were assigned to the low-BMI group (thin).

\section{Stimuli}

Our laboratory has used citric acid to stimulate sAA since 1978. We used our standard method of stimulation: pieces of filter paper of fixed size $(1 \times 1 \mathrm{~cm})$ were soaked in $0.4 \mathrm{~mol} / 1$ citric acid solution for $10 \mathrm{~min}$, then dried in a drying oven, and finally collected and stored in a small and clean container until their use. 


\section{Saliva collection, handling and storage}

All the participants were invited to the outpatient department of Haizu Maternal and Child Health Hospital on Saturday between 09.00 and 11.00 hours in the morning. During a 30-min resting period to minimise the impact of physical activity and emotions, height $(\mathrm{m})$ and weight $(\mathrm{kg})$ of all the participants were measured. Immediately after the end of the resting period, unstimulated whole saliva was collected by passive drooling in a fashion similar to that described by Navazesh ${ }^{(25)}$. Briefly, participants were instructed to seat with their eyes open and head tilted slightly forward, and to empty their mouths by swallowing all saliva. After that, secreted saliva was allowed to drip off the lower lip into a $5 \mathrm{ml}$ test-tube for $3 \mathrm{~min}$. Immediately after that, stimulated whole saliva was collected by the method described below. Participants were instructed to open their mouths and slightly extend their tongues out, and then a filter paper containing citric acid (described above) was placed on the tongue tip of each participant to stimulate saliva for $1 \mathrm{~min}$, during which the stimulated saliva was collected from under the tongue in a $5 \mathrm{ml}$ test-tube. Participants were required to keep their tongue tips slightly upward when collecting stimulated saliva, so that citric acid in the filter paper would not mix with the collected saliva. The $\mathrm{pH}$ values of all saliva samples were determined before storage using $\mathrm{pH}$ test paper with a range from 6.0 to 8.0. All saliva samples went through one freeze-thaw cycle to break down mucopolysaccharides that could interfere with pipetting ${ }^{(26)}$. Upon thawing at $4^{\circ} \mathrm{C}$, saliva was centrifuged at $11000 \mathrm{~g}$ at $4^{\circ} \mathrm{C}$ for $10 \mathrm{~min}$. Then, the supernatant was aliquoted and stored at $-80^{\circ} \mathrm{C}$ for subsequent measurements of sAA amount and activity, while the remaining precipitate (containing cheek cells) was collected and stored at $-80^{\circ} \mathrm{C}$ for subsequent $A M Y 1$ gene analysis.

\section{SDS-PAGE and immunoblotting for salivary amylase}

sAA amount $(\mu \mathrm{g} / \mathrm{ml})$ was determined by Western blot in a fashion similar to that described by Perry et $a l^{(5)}$. Briefly, saliva total protein was determined using BCA Protein Assay Kit (CoWin Bioscience). Saliva samples of equal quantity of total protein $(5 \mu \mathrm{g})$ were prepared by solubilising samples in SDS-PAGE sample loading buffer and heating at $100^{\circ} \mathrm{C}$ for $5 \mathrm{~min}$. For quantification purpose, a human sAA protein sample (Sigma-Aldrich) of known quantity was run on each gel. Proteins were separated by SDS-PAGE and transferred onto a polyvinylidene fluoride membrane (Roche) in a transfer buffer for $1.5 \mathrm{~h}$ at $200 \mathrm{~mA}$. Membranes were blocked for $2 \mathrm{~h}$ at room temperature in blocking buffer (PBS, 0.1\% Tween-20) with $5 \%$ milk. Membranes were incubated overnight at $4{ }^{\circ} \mathrm{C}$ with rabbit anti- $\alpha$-amylase (Abcam), diluted 1:5000 in blocking buffer without milk. After washing in phosphate-buffered saline Tween-20 (PBST), membranes were incubated for $2 \mathrm{~h}$ in goat anti-rabbit IgG-horseradish peroxidase conjugate (R\&D Systems), diluted 1:1000 in blocking buffer without milk. After washing, the membranes were exposed to 3,3'-diaminobenzidine (DAB) substrate (Tiangen Biotech) for $5 \mathrm{~min}$. A ChemiDocXRS $^{+}$Chemiluminescence system (Bio-Rad) was used for detection of amylase. Quantification of protein bands was performed using Gel-Pro Analyzer 4.0 software (Media Cybernetics). The test sAA amount was estimated by comparing with the human sAA of known quantity.

\section{Enzymatic activity assay for salivary amylase}

Because salivary and pancreatic $\alpha$-amylase share homology of $97 \%$, we measured sAA activity according to the method established for pancreatic $\alpha$-amylase activity assay using a commercially available kinetic reaction assay kit (Kofa Biotech Company) on Hitachi 7180 automatic biochemical analyzer in the clinical laboratory of First Affiliated Hospital of Guangdong Pharmaceutical University. Briefly, diluted saliva (1:200) was incubated with a specific chromogenic substrate, 4,6-ethyliden-G7-PNP, and the auxiliary enzyme $\alpha$-glucosidase. The substrate was cleaved by sAA into intermediate products, which were further broken down by the auxiliary enzyme into $p$-nitrophenol (PNP), which absorbed light at a wavelength of $405 \mathrm{~nm}$ (yellow), and glucose. The resulting colour change was directly proportional to sAA activity, thus the sAA activity was determined by measuring the light absorbance value, and was expressed as units $/ \mathrm{ml}$. Intra- and inter-assay precision expressed as percent CV were below $5 \%$.

\section{DNA extraction and quantitative PCR for the AMY1 gene}

Genomic DNA was extracted from cheek cells by the method described below. Briefly, the frozen salivary precipitate (containing cheek cells) was thawed at $4^{\circ} \mathrm{C}$, and then washed and scattered using 0.01 M-PBS. Cheek cells were collected by centrifugation at $2300 \mathrm{~g}$ for $10 \mathrm{~min}$. Cell lysis was achieved by adding $50 \mu \mathrm{l} 5 \mathrm{~m}$-KI solution and vortexing for $30 \mathrm{~s}$. The lysate was then added with $100 \mu \mathrm{l}$ of $0.9 \% \mathrm{NaCl}$ solution and subsequently $150 \mu \mathrm{l}$ chloroform. After vortexing for $30 \mathrm{~s}$ and then centrifugation at $11000 \mathrm{~g}$ for $3 \mathrm{~min}$, the supernatant was transferred to a new Eppendorf tube, followed by adding equal volume of isopropanol. After centrifugation at $11000 \mathrm{~g}$ for $3 \mathrm{~min}$, the precipitated DNA was washed once by $500 \mu \mathrm{l}$ ethanol. Finally, DNA was dissolved by adding $30 \mu \mathrm{l}$ TE buffer (10 mm-Tris and $1 \mathrm{~mm}$-EDTA) and then stored at $-20^{\circ} \mathrm{C}$.

Quantitative PCR (qPCR) was performed to determine diploid $A M Y 1$ gene copy number by the method described by Perry et $a l .{ }^{(5)}$. A fragment from the tumour protein $\mathrm{p} 53$ (TP53) gene was also amplified to adjust for DNA dilution quantity variation. We used previously published primers for AMY1 and TP53 gene fragment amplification ${ }^{(5)}$. Extracted DNA was quantified using NanoDrop (Thermo Scientific), and concentration of all samples was brought to $10 \mathrm{ng} / \mu \mathrm{l}$. PCR amplifications were carried out in a reaction volume of $20 \mu \mathrm{l}$ on a Bio-Rad CFX96 Touch Real-Time PCR Machine. Each qPCR mixture was composed of $10 \mu \mathrm{l}$ PCR mix (BioRad Super mix SYBR Green), $1 \mu$ l of each amplification primer $(1 \mathrm{pmol} / \mu \mathrm{l}), 7 \mu \mathrm{l}$ PCR-analysed water and $1 \mu \mathrm{l}$ DNA extract $(0.5 \mathrm{ng} / \mu \mathrm{l})$. All samples were run in triplicate. Thermal cycling was organised in three repeated steps: the first denaturation step of $3 \mathrm{~min}$ at $95^{\circ} \mathrm{C}$, followed by thirty-nine repeated cycles of $95^{\circ} \mathrm{C}$ for $15 \mathrm{~s}$ and $60^{\circ} \mathrm{C}$ for $30 \mathrm{~s}$. Melting curves were 
obtained by increasing the temperature from 55 to $90^{\circ} \mathrm{C}$ with a plate reading every $0 \cdot 2^{\circ} \mathrm{C}$. Data were analysed using CFX Manager Software version 2.1 (Bio-Rad). $A M Y 1$ diploid copy number was estimated using a standard curve constructed from the reference DNA sample (NA18972; Coriell Cell Repositories), which was previously determined to have fourteen AMY1 diploid copies by qPCR and Fiber FISH (fluorescence in situ hybridisation) ${ }^{(5)}$.

\section{Statistical analyses}

Data were tested for normal distribution and homogeneity of variance by the Kolmogorov-Smirnov and Levene's tests before statistical analyses were conducted. These analyses revealed normal distribution of all variables in both groups, with the exception of the resting SAA amount, the stimulated sAA amount and the resting sAA activity of the Low-BMI group (but not Normal-BMI group). Therefore, we logtransformed data for the resting sAA amount, the stimulated sAA amount and the resting sAA activity in each group (the Low-BMI and the Normal-BMI) before analyses, which successfully restored normality of distribution. Data were presented as means and standard deviations. Student's $t$ test was used for comparison of means. Relationships between data sets were analysed using the Pearson correlation coefficient. Data analysis was performed by IBM SPSS statistics version 19.0 (SPSS). For all analyses, a $P$ value (two-tailed) of less than 0.05 was considered to be statistically significant.

\section{Results}

\section{Sample characteristics}

The main characteristics of the study groups (Normal-BMI children and Low-BMI children) are summarised in Table 1. The results revealed no significant difference in sex, age, weight, height, flow rate of resting or stimulated saliva, and $\mathrm{pH}$ value of resting or stimulated saliva between the two study groups, while BMI has been shown to be significantly different between them $(t=6.434 ; P<0.0001)$. We recorded complaints of poor appetite in both groups. Two boys and two girls in the Normal-BMI group, and five boys and seven girls in the Low-BMI group reported poor appetite. All the participants were from southern China, and mainly fed on rice (starch-rich food).

\section{Salivary amylase levels}

We observed significant individual variation in sAA amount $(\mu \mathrm{g} / \mathrm{ml})$. For the resting sAA, the average amount of the Normal-BMI group and the Low-BMI group were 63.8 (SD 45.9) and 181.0 (SD 230.6) $\mu \mathrm{g} / \mathrm{ml}$, ranging from 10.8 to 174.2 and $17 \cdot 4$ to $842 \cdot 8 \mu \mathrm{g} / \mathrm{ml}$, respectively. For the stimulated sAA, the average amount of the Normal-BMI group and the Low-BMI group was 121.2 (SD 86.8) and 225.9 (SD $310 \cdot 8) \mu \mathrm{g} / \mathrm{ml}$, ranging from 5.9 to 285.1 and 12.8 to $1064.9 \mu \mathrm{g} / \mathrm{ml}$, respectively. Both resting and stimulated sAA amounts in the Low-BMI group were not normally distributed. Therefore, for statistical analyses, the resting and stimulated sAA amounts of both groups were log-transformed, which restored normal distribution. There were no significant differences between the two study groups in transformed values for resting sAA amount $(t=1.670 ; P=0 \cdot 103$; Fig. 1(a) $)$ and the stimulated sAA amount $(t=0.516 ; P=0.608$; Fig. $1(\mathrm{~b}))$.

We also observed significant individual variation in sAA activity per unit of saliva. For the resting sAA, the average activity per unit saliva of the Normal-BMI group and the LowBMI group was 14.3 (sD 8.3) and 32.4 (sD 33.8) units/ml, ranging from 4.4 to 33.8 and 1.4 to 149 units $/ \mathrm{ml}$, respectively. For the stimulated SAA, the average activity per unit saliva of the Normal-BMI group and the Low-BMI group was $32 \cdot 2$ (SD 21.3) and 36.1 (SD 19.6) units/ml, ranging from 6 to $77 \cdot 8$ and 1.6 to 66.8 units $/ \mathrm{ml}$, respectively. Resting sAA activity in the Low-BMI group was not normally distributed. Therefore, for statistical analyses, the resting sAA activity of both groups was log-transformed, which restored normal distribution. There was no significant difference between the two groups in the transformed values for resting sAA activity $(t=1.838$; $P=0.076$; Fig. 1(c)). We also observed no significant difference between the two groups in stimulated sAA activity $(t=0.632 ; P=0.531$; Fig. $1(\mathrm{~d}))$. Together, these results indicate that sAA levels (resting or stimulated) are not directly associated with nutritional status of children.

Table 1. Sample characteristics of normal-BMI and low-BMI children

(Mean values, standard deviations and ranges)

\begin{tabular}{|c|c|c|c|c|c|c|}
\hline \multirow[b]{2}{*}{ Variables } & \multicolumn{3}{|c|}{ Normal-BMI children ( $n$ 22) } & \multicolumn{3}{|c|}{ Low-BMI children ( $n$ 21) } \\
\hline & Mean & SD & Range & Mean & SD & Range \\
\hline \multicolumn{7}{|l|}{ Sex } \\
\hline Boys & & 13 & & & 10 & \\
\hline Girls & & 9 & & & 11 & \\
\hline Age (years) & $7 \cdot 7$ & 1.8 & $5-11.5$ & $7 \cdot 6$ & $2 \cdot 3$ & $5-12$ \\
\hline Weight $(\mathrm{kg})$ & $22 \cdot 3$ & $5 \cdot 1$ & $16-35$ & $19 \cdot 7$ & $4 \cdot 2$ & $15-27$ \\
\hline Height $(\mathrm{cm})$ & $121 \cdot 6$ & 11.5 & $107-152$ & $120 \cdot 5$ & $11 \cdot 3$ & $103-140$ \\
\hline $\mathrm{BMI}^{*}\left(\mathrm{~kg} / \mathrm{m}^{2}\right)$ & 14.9 & 0.8 & $13 \cdot 3-16 \cdot 6$ & 13.4 & 0.6 & $12 \cdot 2-15 \cdot 2$ \\
\hline Resting saliva flow rate $(\mathrm{ml} / \mathrm{min})$ & 0.18 & 0.11 & $0.03-0.40$ & 0.13 & 0.08 & $0.03-0.37$ \\
\hline Stimulated saliva flow rate $(\mathrm{ml} / \mathrm{min})$ & 0.90 & 0.54 & $0.10-2 \cdot 00$ & 0.85 & 0.46 & $0 \cdot 22-2 \cdot 10$ \\
\hline Resting saliva $\mathrm{pH}$ value & $6 \cdot 7$ & 0.3 & $6 \cdot 4-7 \cdot 1$ & $6 \cdot 8$ & 0.2 & $6 \cdot 4-7 \cdot 1$ \\
\hline Stimulated saliva $\mathrm{pH}$ value & $7 \cdot 0$ & 0.2 & $6 \cdot 6-7 \cdot 3$ & $6 \cdot 9$ & 0.2 & $6 \cdot 4-7 \cdot 2$ \\
\hline
\end{tabular}

${ }^{*} P<0.0001$ ( $t$ test) . 

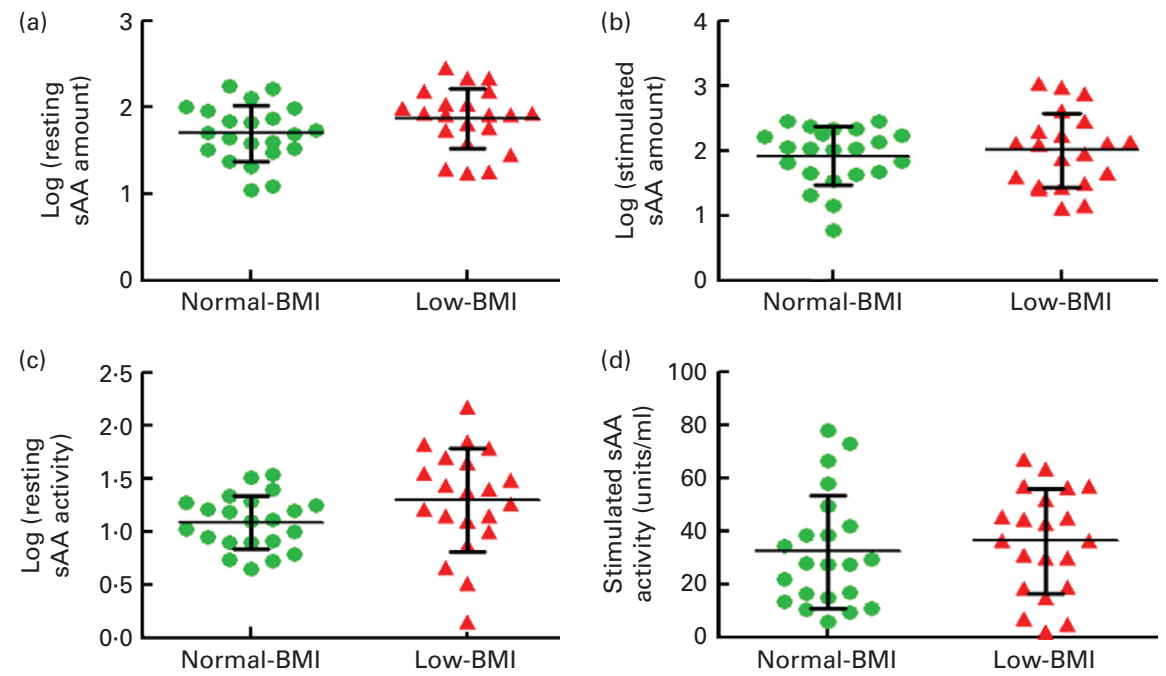

Fig. 1. Comparisons of the resting (a) and stimulated (b) salivary $\alpha$-amylase (SAA) amount, and the resting (c) and stimulated (d) SAA activity between Normal$\mathrm{BMI}$ and Low-BMI children. For statistical tests, the values for resting and stimulated SAA amount, and the resting sAA activity were log-transformed before analyses, which successfully restored normality of distribution. Values are means, with standard deviations represented by vertical bars. No significant difference was observed in log-transformed values for resting SAA amount, stimulated SAA amount, resting sAA activity or stimulated sAA activity between the two study groups. A colour version of this figure can be found online at http://www.journals.cambridge.org/bjn

We also analysed the effects of sex and age on sAA level and found that neither sex nor age were significantly related to sAA levels (resting or stimulated) within or between groups (data not shown).

Because sAA levels have high variability in both previous studies and in the present study, and because individuals are accustomed to their own sAA levels, it is difficult to link sAA levels (resting or stimulated) with individual's nutritional status. We hypothesised that the SAA ratio (the ratio of the stimulated sAA level to that of the resting sAA) may reflect individual's response pattern related to nutritional status. Thus, in the present study, we calculated sAA ratio and analysed its relationship with children's nutritional status. Consistent with our expectation, sAA amount ratio in the Low-BMI children was significantly lower than in the Normal-BMI children $(t=2 \cdot 727 ; P=0 \cdot 009$; Fig. 2(a)). The average sAA amount ratios in the Normal-BMI group and the Low-BMI group were $2 \cdot 0$ (SD 1.2) and $1 \cdot 1$ (SD 0.7), ranging from 0.5 to 5.6 and 0.3 to $2 \cdot 7$, respectively. We also observed
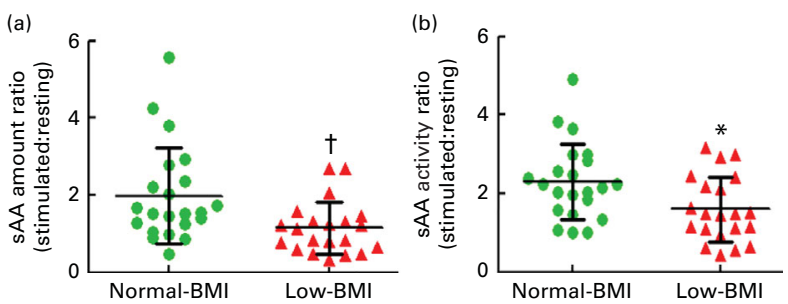

Fig. 2. Comparisons of salivary $\alpha$-amylase (sAA) amount ratio (a) and sAA activity ratio (b) between Normal-BMI and Low-BMI children. SAA amount: activity ratio was expressed as the ratio of stimulated SAA amount:activity to the resting SAA amount:activity. Values are means, with standard deviations represented by vertical bars. *Mean values was significantly different between the two study groups for the sAA amount ratio $(P=0.009)$. † Mean values was significantly different between the two study groups for the sAA activity ratio $(P=0.016)$. A colour version of this figure can be found online at http://www.journals.cambridge.org/bjn a significantly lower sAA activity ratio in the Low-BMI group than in the Normal-BMI group $(t=2 \cdot 522 ; P=0.016$; Fig. 2(b)). The average sAA activity ratio of the Normal-BMI group and the Low-BMI group was 2.3 (SD 1.0 ) and 1.6 (SD 0.8 ), ranging from 1.0 to 4.9 and 0.4 to $3 \cdot 2$, respectively. These results together suggest attenuated acute sAA responses in Low-BMI children. In line with our expectation, the extent to which sAA respond to gustatory stimulation may be of nutritional importance, at least in children.

\section{AMY1 gene copy number}

DNA samples were analysed by qPCR to determine gene copy number. Values were standardised to a human DNA sample with a known $A M Y 1$ gene copy number verified by Fiber FISH. The average number of $A M Y 1$ gene copies of all the participants was 7.8 (SD 2.4). In Normal-BMI and Low-BMI children, the number was 7.6 (SD 2.7) and 8.0 (SD 2.1), ranging from 3 to 13 and 3 to 11 , respectively. No significant difference was observed in $A M Y 1$ gene copy number between the two study groups ( $t=0.43 ; P=0.670$ ) (Fig. 3 ). Therefore, $A M Y 1$ $\mathrm{CNV}$ is not directly associated with child's nutritional status.

We next analysed the relationships between $A M Y 1 \mathrm{CNV}$ and sAA levels in children. Because previous studies have shown that sAA concentration reflects the combined effects of protein secretion and salivary flow rate ${ }^{(27)}$, in the present study, we made correction for salivary flow rate when assessing the association between sAA levels (concentration and activity) with CNV of $A M Y 1$. We did not observe a significant correlation between $A M Y 1$ gene copy number and sAA concentration $(r 0.013 ; P=0.934)$ or activity $(r 0.029 ; P=0.854)$ in a combined group including all the participants. We also did not observe significant correlations within Normal-BMI group $((r) \cdot 180 ; P=0.422)$ for sAA concentration and CNV of $A M Y 1 ;(r \quad 0 \cdot 117 ; P=0.604)$ for sAA activity and CNV of 


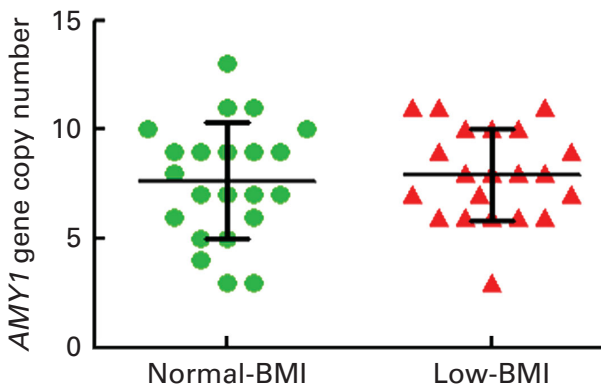

Fig. 3. Comparison of $A M Y 1$ (salivary amylase gene) gene copy number between Normal-BMI and Low-BMI children. Values are means, with standard deviations represented by vertical bars. No significant difference between the two study groups was observed in $A M Y 1$ gene copy numbers. A colour version of this figure can be found online at http://www.journals. cambridge.org/bjn

$A M Y 1)$ or Low-BMI group $((r 0.060 ; P=0.796)$ for sAA concentration and CNV of $A M Y 1 ;(r \quad 0.014 ; P=0.622)$ for sAA activity and $\mathrm{CNV}$ of $A M Y 1$ ). Our observations suggest that AMY1 CNV may influence but do not eventually determine sAA levels in children.

\section{Discussion and conclusion}

The present study, for the first time, found attenuated acute sAA responses induced by citric acid in Low-BMI children (thinness grade 3) compared with Normal-BMI children. This finding suggests that sAA responses to gustatory stimulation are of nutritional importance in children. However, we found no relationships of resting or stimulated sAA levels with nutritional status. We also did not observe any associations between $A M Y 1$ gene copy number and child's nutritional status. Finally, $A M Y 1 \mathrm{CNV}$ might influence but did not eventually determine sAA levels in children.

Because sAA is increased during stress, i.e. when autonomic nervous system is activated, SAA has been proposed as a marker for activity of the sympathetic nervous system ${ }^{(28)}$. Nevertheless, Bosch et $a l .{ }^{(27)}$ considered the idea as too simple, and argued that the parasympathetic nerves also play a significant role in SAA release via several ways, and that the methodology of supporting publications was problematic. This remains to be further clarified. In the present study, however, we focused our attention on reconsidering the main property of sAA, namely its digestive action towards starch-based food and the consequences related to health.

Starch-based foods (e.g. rice) are considered a dominating energy source for those who traditionally feed on them, and thus influence or even determine their nutritional status. Attempts have been made to link sAA levels (resting and stimulated) with individual's preference for a starchy food $^{(6,15,16)}$. In these studies, researchers found that sAA levels were associated with individual's oral perception of textural attributes of starchy foods. These findings may explain how sAA relates to individual's nutritional status, especially for those who traditionally feed on starch-rich food. These linkages, however, seemed to be not so convincing to come to a conclusion. In humans, sweet taste is a powerful factor influencing food choice and consumption; thus, it may affect individual nutrition. Sugars (e.g. maltose and glucose) are recognised by human's taste system (by interactions with sweet taste receptors expressed on the taste buds) and evoke appetitive consummatory responses ${ }^{(29)}$. In the oral cavity, starch-based food is partially hydrolysed by sAA into maltose and glucose after mastication. Both these sugars can act as sweet taste stimuli to interact with taste receptor type 1 (T1R) 2-T1R3 sweet taste receptor, and then evoke pleasant sweet perception and influence ingestive behaviour ${ }^{(30)}$. In addition to evoking behavioural responses, sweet taste stimuli can elicit preabsorptive cephalic phase responses. For instance, oral glucose can induce a significant preabsorptive elevation of insulin levels, known as cephalic-phase insulin release (CPIR), in rats ${ }^{(31)}$ and humans ${ }^{(18)}$. CPIR plays an extremely important role in glycaemic homeostasis ${ }^{(32)}$, and thus can affect individual nutrition. Interestingly, CPIR was significantly different between high sAA activity and low sAA activity individuals after starch ingestion but not after glucose ingestion $^{(18)}$, which suggest that CPIR following starch ingestion is dependent on sAA level. Sweet taste stimuli, moreover, can activate endogenous dopaminergic and serotonergic systems $^{(33,34)}$, which play a crucial role in neural basis for food intake and appetite ${ }^{(35,36)}$. Collectively, the way how sAA levels are related to individual nutritional status is probably following: sAA levels influence the break down of starch-based foods into maltose and glucose in the oral cavity; then the two sugars affect individual sweet taste perception, CPIR, and endogenous dopaminergic and serotonergic systems, which together determine starch-based food consumption (energy intake) and eventually influence individual nutritional status.

Starch-based food intake generally involves mastication, which preferentially stimulates output of $\mathrm{SAA}^{(37)}$. Stimulated sAA might influence individual's appetite ${ }^{(38)}$. Because sAA levels show high variability among or within individuals, and because individuals are accustomed to their own idiosyncratic salivary flow rates and sAA levels, we hypothesised that the degree to which sAA respond to food intake is thus expected to be of nutritional importance. In line with our expectation, we observed a significantly decreased sAA ratio in Low-BMI children when compared with that of NormalBMI children. In the present study, we received more complaints of poor appetite in the Low-BMI group from children themselves or their parents. Based on the above discussion, the decreased sAA ratio seems to be partially responsible for the poor appetite: the decreased sAA levels may influence individual sweet taste perception, CPIR, and endogenous dopaminergic and serotonergic systems, which together decrease appetite for, and consumption of, the starch-based foods in Low-BMI children. Complaints from parents might be due to their high expectation for children to eat; however, taken together, we had reasons to believe that the Low-BMI children show poorer appetite than did the Normal-BMI children did. There were concerns that a chronic restriction of food intake may have a long-term effect on sAA secretion ${ }^{(39)}$. However, Rohleder and Nater argued that this effect might be pronounced when examined on an evolutionary time scale $^{(19)}$. In addition, the present study investigated acute sAA 
responses induced by citric acid, and did not find significant difference in resting or stimulated sAA levels between Normal-BMI and Low-BMI children. Therefore, we argued that the decreased SAA ratio might be partially responsible for the poorer appetite, which, in turn, influenced individual's nutritional status in the Low-BMI children.

In the present study, we found higher number of $A M Y 1$ gene copies (average of 7.8 copies) than in previous studies with low-starch populations with the average number of copies $5^{(5)}$ and $4 \cdot 4^{(6)}$. This suggests that a significant proportion of our subjects' ancestors may undergo positive selection for increased $A M Y 1$ gene copy number, rather than this gene CNV evolving neutrally. A significant variation in sAA levels, however, was not explained by $A M Y 1$ gene copy numbers in the present study. This lack of relationship might be explained by the following: (1) sAA expression might reflect other genetic influences, such as regulatory region SNP in the $A M Y 1$ gene or differences in the transcription or translation efficiency between $A M Y 1$ genes in different haplotypes ${ }^{(4,5)}$; (2) nongenetic influences (e.g. physical activity and psychosocial stress conditions) on sAA secretion; (3) protein modifications (e.g. N-glycosylation), which affect enzyme activity ${ }^{(40)}$. Moreover, we did not observe significant correlation between AMY1 gene copy number and sAA levels in children, while previous work has shown significant correlation in adults ${ }^{(6)}$. This might be due to subjects' age difference. Development of human salivary glands is morphologically completed in utero; however, they continue to grow during childhood by proliferation of well-differentiated cells ${ }^{(41)}$, and salivary glands function and saliva compositions change with aging ${ }^{(42)}$.

Mandel et $a l^{(6)}$ failed to observe a strong relationship between sAA concentration and the enzymatic activity. Note that the variation in sAA levels in the present study was even more pronounced than in the previous studies, which might be due to age-related and ethnical differences. The present study included children who have higher baseline sAA levels than adults ${ }^{(20)}$. In addition, all the participants were Chinese whose ancestors traditionally fed on rice starting from thousands years ago, and thus are generally categorised as a high-starch consuming population.

In the present study, we did not observe associations between $A M Y 1 \mathrm{CNV}$ and child's nutritional status, which might be explained by the following reasons. First, child's nutritional status is more likely to be determined by combined action of various genes, rather than by a single gene. Second, because children are in a sensitive period of growth and development, they may be more susceptible to environmental changes than to genetic factors. For example, changes in oral perception may significantly influence child's liking and preference for foods, and thus play a crucial role in determining child's nutritional status. Finally, $A M Y 1 \mathrm{CNV}$ and individual nutritional status are the most distal levels of analysis in the study; it is not surprising we failed to find a connection between them.

We stimulated saliva production by applying citric acid to the tongue. If citric acid would mix with saliva, this would change salvia $\mathrm{pH}$ value, and thus would interfere with assay results. However, we designed our technique to prevent this from occurring. Our laboratory has successfully applied citric acid to stimulate sAA for more than 30 years by using the standardised method described in the Materials and methods section. As a quality control, we measured $\mathrm{pH}$ of saliva samples, and a saliva sample would be discarded if its $\mathrm{pH}$ value was less than $6 \cdot 4$, which was considered to be the lower limit of saliva $\mathrm{pH}$ value. We reported that no saliva samples were less than 6.4 as for $\mathrm{pH}$ value in the present study.

The present study has two potential limitations. The first one is a relatively small sample size. Although we could have easily recruited more Normal-BMI children, it was difficult to find Low-BMI children in South China. The second limitation is our ability to accurately evaluate child's appetite. Children typically have difficulties in describing their own appetite partially due to their ignorance, and their parents may have excessively high expectations for their children to eat; together, this may have contributed to variations in evaluating appetite. Thus, findings of the present study should be considered as preliminary until replicated in future research with larger sample sizes.

In conclusion, the present study, for the first time, demonstrated attenuated sAA responses to gustatory stimulation in thin (Low-BMI) children. This suggests that sAA responses to gustatory stimulation may affect individual's nutritional status. $A M Y 1 \mathrm{CNV}$ was not associated with child's nutritional status in our sample. Finally, $A M Y 1 \mathrm{CNV}$ might partially influence but did not eventually determine sAA levels in children.

\section{Acknowledgements}

The authors thank all the study participants. They also thank Dr Alexander Bachmanov, Monell Chemical Senses Center, Philadelphia, USA, for his critical review of the present study. The authors also thank Dr Chuan Wei Mo, Guangzhou University of Chinese Medicine, Guangzhou, China, for his assistance in the statistical analyses.

The present study was supported by the National Natural Science Foundation of China (Z. M. Y., grant no. 81102703), the Administration of Traditional Chinese Medicine of Guangdong Province of China (Z. M. Y., grant no. 20123001), the Science and Technology Planning Project of Guangdong Province of China (Z. M. Y., grant no. 2013A032500005) and the Special Funds from Central Finance of China in Support of the Development of Local Colleges and Universities in 2013 (W. W. C., grant no. 338). The funders had no role in the study design, data collection and analysis of the study, in the decision to publish, or in the preparation of the manuscript.

The authors' contributions are as follows: L. H. C. wrote the manuscript, collected data and assisted with the Western blot and qPCR experiments; Z. M. Y. and W. W. C. were the principal investigators and together contributed to the development of the overall research plan, study protocol and study oversight, and analysed the data; J. L. assisted with the Western blot and qPCR experiments; M. Z. was responsible for the participant recruitment, physical examination and saliva sample collection; X. R. Y. performed the enzymatic activity assay; L. B. Z. assisted with participant recruitment and saliva sample collection.

None of the authors has any conflicts of interest. 


\section{References}

1. Oppenheim FG, Salih E, Siqueira WL, et al. (2007) Salivary proteome and its genetic polymorphisms. Ann $N$ Y Acad Sci 1098, 22-50.

2. Noble RE (2000) Salivary $\alpha$-amylase and lysozyme levels: a non-invasive technique for measuring parotid vs submandibular/sublingual gland activity. J Oral Sci 42, 83-86.

3. Lebenthal E (1987) Role of salivary amylase in gastric and intestinal digestion of starch. Dig Dis Sci 32, 1155-1157.

4. Bank RA, Hettema EH, Muijs MA, et al. (1992) Variation in gene copy number and polymorphism of the human salivary amylase isoenzyme system in Caucasians. Hum Genet 89, 213-222.

5. Perry GH, Dominy NJ, Claw KG, et al. (2007) Diet and the evolution of human amylase gene copy number variation. Nat Genet 39, 1256-1260.

6. Mandel AL, Peyrot des Gachons C, Plank KL, et al. (2010) Individual differences in $A M Y 1$ gene copy number, salivary $\alpha$-amylase levels, and the perception of oral starch. PLoS ONE 5, e13352.

7. Groot PC, Bleeker MJ, Pronk JC, et al. (1989) The human $\alpha$-amylase multigene family consists of haplotypes with variable numbers of genes. Genomics 5, 29-42.

8. Iafrate AJ, Feuk L, Rivera MN, et al. (2004) Detection of largescale variation in the human genome. Nat Genet 36, 949-951.

9. Chatterton RT Jr, Vogelsong KM, Lu YC, et al. (1996) Salivary $\alpha$-amylase as a measure of endogenous adrenergic activity. Clin Physiol 16, 433-448.

10. Nater UM, Rohleder N, Gaab J, et al. (2005) Human salivary $\alpha$-amylase reactivity in a psychosocial stress paradigm. Int $J$ Psychophysiol 55, 333-342.

11. Nater UM, La Marca R, Florin L, et al. (2006) Stress-induced changes in human salivary $\alpha$-amylase activity - associations with adrenergic activity. Psychoneuroendocrinology 31, 49-58.

12. Behall KM, Kelsay JL, Holden JM, et al. (1973) Amylase and protein in parotid saliva after load doses of different dietary carbohydrates. Am J Clin Nutr 26, 17-22.

13. Messenger B, Clifford MN \& Morgan LM (2003) Glucosedependent insulinotropic polypeptide and insulin-like immunoreactivity in saliva following sham-fed and swallowed meals. J Endocrinol 177, 407-412.

14. Froehlich DA, Pangborn RM \& Whitaker JR (1987) The effect of oral stimulation on human parotid salivary flow rate and $\alpha$-amylase secretion. Physiol Behav 41, 209-217.

15. de Wijk RA, Prinz JF, Engelen L, et al. (2004) The role of $\alpha$-amylase in the perception of oral texture and flavour in custards. Physiol Behav 83, 81-91.

16. Engelen L, van den Keybus PA, de Wijk RA, et al. (2007) The effect of saliva composition on texture perception of semi-solids. Arch Oral Biol 52, 518-525.

17. Prindiville EA, Marshall RT \& Heymann H (2000) Effect of milk fat, cocoa butter, and whey protein fat replacers on the sensory properties of lowfat and nonfat chocolate ice cream. J Dairy Sci 83, 2216-2223.

18. Mandel AL \& Breslin PA (2012) High endogenous salivary amylase activity is associated with improved glycemic homeostasis following starch ingestion in adults. J Nutr 142, 853-858.

19. Rohleder N \& Nater UM (2009) Determinants of salivary $\alpha$-amylase in humans and methodological considerations. Psychoneuroendocrinology 34, 469-485.

20. Strahler J, Mueller A, Rosenloecher F, et al. (2010) Salivary $\alpha$-amylase stress reactivity across different age groups. Psychophysiology 47, 587-595.

21. de Campos MM, Kobayashi FY, Barbosa TD, et al. (2013) Characteristics of salivary secretion in normal-weight, overweight and obese children: a preliminary study: salivary composition and excessive fat tissue. Odontology 102, 318-324.

22. Cole TJ, Flegal KM, Nicholls D, et al. (2007) Body mass index cut offs to define thinness in children and adolescents: international survey. BMJ 335, 194.

23. Group of China Obesity Task Force (2004) Body mass index reference norm for screening overweight and obesity in Chinese children and adolescents. Chin J Epidemiol 25, 97-102.

24. Bishop NC, Walker GJ, Scanlon GA, et al. (2006) Salivary IgA responses to prolonged intensive exercise following caffeine ingestion. Med Sci Sports Exerc 38, 513-519.

25. Navazesh M (1993) Methods for collecting saliva. Ann $N Y$ Acad Sci 694, 72-77.

26. Shirtcliff EA, Granger DA, Schwartz E, et al. (2001) Use of salivary biomarkers in biobehavioral research: cotton-based sample collection methods can interfere with salivary immunoassay results. Psychoneuroendocrinology 26, 165-173.

27. Bosch JA, Veerman EC, de Geus EJ, et al. (2011) $\alpha$-Amylase as a reliable and convenient measure of sympathetic activity: don't start salivating just yet! Psychoneuroendocrinology 36, 449-453.

28. Nater UM \& Rohleder N (2009) Salivary $\alpha$-amylase as a noninvasive biomarker for the sympathetic nerous system: current state of research. Psychoneuroendocrinology 34, 486-496.

29. McCaughey SA (2008) The taste of sugars. Neurosci Biobehav Rev 32, 1024-1043.

30. Bachmanov AA, Bosak NP, Floriano WB, et al. (2011) Genetics of sweet taste preferences. Flavour Fragr J 26, 286-294.

31. Grill HJ, Berridge KC \& Ganster DJ (1984) Oral glucose is the prime elicitor of preabsorptive insulin secretion. $A m J$ Physiol 246, R88-R95.

32. Ahrén B \& Holst JJ (2001) The cephalic insulin response to meal ingestion in humans is dependent on both cholinergic and noncholinergic mechanisms and is important for postprandial glycemia. Diabetes 50, 1030-1038.

33. O'Doherty JP, Deichmann R, Critchley HD, et al. (2002) Neural responses during anticipation of a primary taste reward. Neuron 33, 815-826.

34. Cooper SJ \& Barber DJ (1994) Evidence for serotonergic involvement in saccharin preference in a two-choice test in rehydrating rats. Pharmacol Biochem Behav 47, 541-546.

35. Baik JH (2013) Dopamine signaling in reward-related behaviors. Front Neural Circuits 7, 152.

36. Halford JC \& Blundell JE (2000) Separate systems for serotonin and leptin in appetite control. Ann Med 32, 222-232.

37. Navazesh M \& Kumar SK (2008) Measuring salivary flow: challenges and opportunities. J Am Dent Assoc 139, 35S-40S.

38. Harthoorn LF \& Dransfield E (2008) Periprandial changes of the sympathetic-parasympathetic balance related to perceived satiety in humans. Eur J Appl Physiol 102, 601-608.

39. Schumacher S, Kirschbaum C, Fydrich T, et al. (2013) Is salivary $\alpha$-amylase an indicator of autonomic nervous system dysregulations in mental disorders? - a review of preliminary findings and the interactions with cortisol. Psychoneuroendocrinology 38, 729-743.

40. de Barros MC, do Nascimento Silva R, Ramada MH, et al. (2009) The influence of N-glycosylation on biochemical properties of Amy1, an $\alpha$-amylase from the yeast Cryptococcus flavus. Carbohydr Res 344, 1682-1686.

41. Redman RS (2008) Development of the salivary glands. In The Salivary System, pp. 1-20 [LM Sreebny, editor]. Boca Raton, FL: CRC Press.

42. Ghezzi EM \& Ship JA (2003) Aging and secretory reserve capacity of major salivary glands. J Dent Res $\mathbf{8 2}$, 844-848. 